\title{
Influência da família na primeira fase de desenvolvimento da carreira de nadadores medalhistas olímpicos brasileiros
}

\author{
Family influence in the first phase of the career development of Brazilian \\ swimmers Olympic medalists
}

\author{
R.M. Ferreira, L.C. Moraes
}

ARTIGO ORIGINAL | ORIGINAL ARTICLE

\begin{abstract}
A presença familiar é fundamental para o apoio ao atleta em todos os estágios de desenvolvimento, principalmente nos períodos de transição da carreira dos atletas. O objetivo do presente estudo foi analisar o papel que a família de nadadores medalhistas olímpicos brasileiros tem em relação aos tipos de apoio / influências durante a primeira fase de desenvolvimento de suas carreiras. Oito nadadores, que conquistaram pelo menos uma medalha olímpica, compuseram esta amostra. Foi utilizado um roteiro de entrevista semiestruturada retrospetiva de aprofundamento. Os dados foram analisados por meio de Meaning Units (Mini-Unidades - MUs). Os resultados indicam que durante o início de suas carreiras, os atletas receberam de suas famílias, principalmente, apoio emocional e financeiro. Mesmo se tratando de um levantamento histórico, conclui-se que a presença da família representa um pilar fundamental para o desenvolvimento da maioria dos nadadores analisados.

Palavras-chave: nadadores olímpicos, família, desenvolvimento
\end{abstract}

ABSTRACT

Family presence and support are extremely important factors for an athlete at all stages of development, especially during the transitional stages of the athlete's career. The objective of this study was to analyze the role of the family for competitive swimmers in relation to the types of support and/or influence those families have during the first stage of the athlete's career. Eight swimmers who have won an Olympic medal composed this study. A semi-structured interview script requiring deep retrospective answers from the athletes was used. The data was analyzed using Meaning Units (MUs). Results show that during the beginning of their careers, the athletes received mostly emotional and financial support from their families. Even though this is an historic study, it was concluded that the presence of the family represents a fundamental role in the development of the majority of the swimmers analyzed.

Keywords: Olympic swimmers, family, development

Submetido: 01.05.2010 | Aceite: 19.04.2011

Renato Melo Ferreira e Luiz Carlos de Moraes. Escola de Educação Física, Fisioterapia e Terapia Ocupacional EEFFTO; Universidade Federal de Minas Gerais - UFMG, Minas Gerais, Brasil.

Endereço para correspondência: Renato Melo Ferreira, Escola de Educação Física, Fisioterapia e Terapia Ocupacional - EEFFTO, Universidade Federal de Minas Gerais - UFMG, Av. Presidente Antônio Carlos, 6627, CENESP - LAPES - Pampulha, CEP: 31270-901 - Belo Horizonte, MG, Brasil

E-mail: renato.mf@hotmail.com 
Estudos que analisam o desenvolvimento de jovens expoentes em várias atividades, inclusive as desportivas, comprovam que a presença familiar é fundamental para o apoio ao atleta, principalmente nos períodos de transição das suas carreias (Bloom, 1985; Côté, 1999; Durand-Bush \& Salmela, 2002; Moraes, Salmela, Rabelo, \& Vianna Jr, 2004b; Salmela \& Moraes, 2003; Salmela, Young, \& Kallio, 2000; Moraes, Rabelo, \& Salmela, 2004a). Todos os trabalhos supracitados elucidam o papel fundamental que os pais exercem sobre os atletas, essencialmente nos anos iniciais (Bloom, 1985), pois, o adequado envolvimento destes irá possibilitar o progresso do atleta (Hellstedt, 1987). Bloom (1985) investigou, num estudo retrospetivo, a vida de expoentes em diversas áreas. O seu trabalho, plurianual, contou com pianistas, nadadores olímpicos, tenistas e escultores, entre outros. Os resultados indicaram que a qualidade do apoio da família foi um fator preponderante para o progresso em direção ao alto desempenho nas suas áreas. Bloom sugeriu ainda três estágios distintos (anos iniciais, anos intermediários e anos finais) que marcam o processo de desenvolvimento da criança nesses campos.

Nos anos iniciais, os pais são responsáveis por inserir os seus filhos numa modalidade desportiva, sendo que o apoio emocional e financeiro é fundamental para que a criança se mantenha praticante (Bloom, 1985; Moraes, Rabelo, \& Salmela, 2004a). Nos anos intermediários, os pais contribuem com o apoio financeiro para despesas com materiais, viagens e melhores condições de treino, sendo determinantes para o desenvolvimento dos seus filhos. Tal apoio garante a manutenção da atividade desportiva dos filhos em relação aos mais variados aspetos, como os motivacionais (Vernacchia, Mcguire, Reardon, \& Templin, 2000), a realização, o comprometimento (Mallet \& Hanrahan, 2004) e a prática. Nos anos finais, muitas das vezes, as famílias mudam para outra cidade a fim de proporcionarem melhores condições de treino para os filhos (Marques \& Samulski, 2009).
Côté (1999), ao realizar um estudo similar ao desenvolvido por Bloom, investigou o processo de desenvolvimento de expoentes no desporto, mais especificamente em relação à influência das suas famílias. Os resultados, desse estudo retrospetivo, resultaram, também, na identificação de estágios de desenvolvimento dos atletas no desporto, caracterizados como: anos de experimentação, de especialização, de investimento e de manutenção e aperfeiçoamento.

Nos anos de experimentação (6 a 13 anos), destacou-se o apoio dos pais e treinadores, sendo estes fundamentais para o desenvolvimento da criança na modalidade durante esse período. Nos anos de especialização (13 a 15 anos), o apoio dos pais ainda continuou presente, principalmente o financeiro. A presença de modelos dentro de casa, como irmãos mais velhos, que também foram atletas, constituiu uma referência importante para a persistência dos filhos no desporto. Nos anos de investimento (dos 15 anos em diante), a família ainda apoiou financeira e emocionalmente o futuro atleta. Já no último estágio, nos anos de perfeição, a ajuda disponibilizada pelos pais caracterizou-se por um apoio mais emocional.

Durante todos os estágios de desenvolvimento, a relação existente entre pais e filhos é fundamental para o desenvolvimento contínuo das suas carreiras. Dessa forma, Czikszentmihalyi, Rathunde, \& Whalen (1993) estabeleceram dois processos nomeados de integração e diferenciação. As famílias que promovem a integração desenvolvem um sentimento de apoio e harmonia nos filhos e por meio da convivência no lar. Já as famílias que promovem diferenciação, encorajam os seus filhos durante os desafios do processo de formação de competências em determinada área, incluindo a do desporto. Tais famílias visam à independência dos filhos. Além disso, os autores apresentam o conceito de famílias complexas, que são aquelas que promovem tanto a diferenciação quanto a integração, proporcionando as melhores condições de desenvolvimento de crianças e jovens (Durand-Bush \& 
Salmela, 2002; Moraes, Rabelo, Salmela, Lima, \& Lôbo, 2001; Moraes \& Sousa, 2004; Salmela \& Moraes, 2003). Vianna, Moraes, Salmela e Mourthé (2001) realizaram um trabalho com atletas de ginástica rítmica, os resultados demonstraram a existência de um envolvimento efetivo dos pais durante todos os estágios, no entanto, o foco de estudo foi a primeira fase de desenvolvimento. Os pais apoiaram as atletas por meio de recursos financeiros, na busca de melhores condições de treino, e na motivação extrínseca de suas filhas. Todavia, a cobrança, a pressão pelo resultado a qualquer custo, por parte da família, pode levar a criança ao abandono precoce da prática desportiva (Gustafsson, Kenttä, Hassmén, \& Lundqvist, 2007).

Hellstedt (1987) estabeleceu em três níveis o conceito de envolvimento dos pais no desporto; (i) o sub envolvimento, traduzido pela falta de comprometimento financeiro e emocional dos pais, como a ausência nas competições; (ii) o envolvimento adequado, no qual os pais oferecem todo o suporte e ajuda aos filhos em relação ao estabelecimento de metas reais, além de os apoiarem financeiramente, e; (iii) o super envolvimento, onde os pais exageram na sua participação, não sabendo diferenciar as suas necessidades daquelas dos seus filhos.

Portanto, os pais foram apontados como fator determinante para se atingir o sucesso em determinada área, na medida em que proviam o apoio emocional adequado, bem como suporte financeiro e estrutural aos filhos (Baker \& Horton, 2004; Falk, Lidor, Lander, \& Lang, 2004; Ericsson, Prietula, \& Cokley, 2007; Côté, Lidor, \& Hackfort, 2009). Este contexto contribuiu para a atração dos filhos pelo desporto, passando pela escolha da modalidade, pelo desenvolvimento na iniciação desportiva até chegar à participação em competições de alto nível (Bloom, 1985; Côté, 1999).

Moraes et al. (2004b) analisaram a influência da família em crianças praticantes de futebol e os resultados apontaram que, nem sempre, o apoio da família, financeiro ou emocional, interferiu no desenvolvimento dos atletas. Pois, nestes casos, a motivação desses atletas era intrínseca, movidos pela paixão pelo desporto, pelo baixo poder aquisitivo dessas famílias e, principalmente, pela oportunidade de mudança de vida (financeira) que o futebol proporciona. Contudo, essas famílias ofereciam um apoio indireto, já que os jovens atletas eram poupados dos trabalhos nas respetivas casas para poderem jogar futebol no clube local. McCarthy e Jones (2007) realizaram um estudo qualitativo com 45 crianças, no qual o objetivo era examinar quais as fontes que proporcionavam o prazer e o desprazer em praticar atividades desportivas nos anos iniciais de desenvolvimento. Os principais resultados indicaram que as fontes prazerosas para os praticantes eram: o envolvimento social, a relação com os amigos e um bom treinador; já as principais fontes de desprazer foram as cobranças excessivas dos pais e o feedback negativo.

Este trabalho se justifica por, inicialmente, se tratar de um levantamento histórico, que analisa a influência que as famílias dos nadadores medalhistas olímpicos brasileiros tiveram durante o início de suas carreiras, o que pode ter determinado sua permanência na natação. Por conseguinte, o estudo sobre os melhores atletas de uma determinada modalidade se justifica pelo fato de colaborar para a melhora daqueles que pretendem aperfeiçoar seu nível (Strauss \& Corbin, 1990; Morgan \& Giacobbi, 2006). Além disso, a visão da realidade que estes indivíduos têm a respeito da influência de suas famílias serve como base para a compreensão dos componentes da expert performance (Ericsson \& Lehmann, 1996).

O objetivo do presente estudo foi analisar o papel que a família de nadadores medalhistas olímpicos brasileiros tem em relação aos tipos de apoio/influências durante a primeira fase de desenvolvimento de suas carreiras.

\section{MÉTODO}

Para a realização do presente estudo foi utilizada uma abordagem qualitativa, de natu- 
reza retrospetiva, pois esta utiliza uma visão mais holística para analisar o fenômeno investigado (Patton, 2002). No caso do presente estudo, o fenômeno é a influência que as famílias de atletas que conquistaram medalhas olímpicas na natação brasileira têm em suas carreiras durante a fase inicial.

\section{Amostra}

A amostra foi composta por oito nadadores expert com idade média de $46.25 \pm 14.22$ anos e com tempo de prática de $11.37 \pm 3.29$ anos. O critério de seleção foi o atleta ter conquistado, pelo menos, uma medalha (ouro, prata ou bronze) em qualquer uma das edições dos Jogos Olímpicos, podendo ser conquistada em provas individuais ou de estafetas. Os nadadores, com a finalidade de se manter o anonimato, foram aleatoriamente distribuídos por A1 (atleta 1), A2 (atleta 2), ... e A8 (atleta 8). Este estudo foi aprovado pelo Comitê de Ética em Pesquisa da Universidade Federal de Minas Gerais sob protocolo número ETIC 195/09.

\section{Instrumentos}

Foram utilizados para a coleta de dados uma ficha demográfica e um roteiro de entrevista semiestruturada (Côté, 1999; Moraes, 1999; Patton, 2002; Côté, Ericsson, \& Law, 2005; Jonhson, et al., 2008).

$\mathrm{O}$ roteiro de entrevista semiestruturada contou perguntas relativas ao envolvimento das famílias na vida desportiva dos atletas, sendo que a ordem inicial das questões é apresentada na Tabela 1.

As vantagens para este tipo de procedimento são: a possibilidade e coletar uma grande variedade de informação; menor número de questões mal entendidas e respostas impróprias, menor quantidade de respostas incompletas e maior controle sobre o ambiente no qual a entrevista é realizada (Patton, 2002).

Nesse tipo de entrevista, os aspetos a serem investigados são estabelecidos previamente pelo pesquisador. As questões são primeiramente listadas, contudo, estas podem-se desviar do curso inicial, dependendo da necessidade e decisão do pesquisador (Côté, 1999; Johnson et al., 2008; Patton, 2002; Tesch, 1990).

Os equipamentos utilizados para realizar as entrevistas foram dois gravadores digitais (Panasonic RR-US450), um notebook (Acer Aspire 3690-2023), além do software Voice Editing Premium Edition 2.0, para edição de arquivos de áudio.

\section{Procedimentos}

Inicialmente foi obtido um apoio institucional da Confederação Brasileira de Desportos Aquáticos (CBDA), que endossou uma carta respaldando o presente estudo. Posteriormente, com o objetivo de compreender a viabilidade do trabalho, foi realizado, via telefone, um primeiro contato com os nadadores, explicando os principais objetivos do estudo.

Posteriormente, as entrevistas foram agendadas de acordo com a disponibilidade dos voluntários, entre julho e outubro de 2009. Nas datas agendadas, foi assinado o Termo de Consentimento Livre e Esclarecido, e realizada a coleta nas suas respetivas cidades de residência, em locais tranquilos e sem interrupções.

Tabela 1

Ordem inicial das perguntas da entrevista semiestruturada

\begin{tabular}{l}
\hline Perguntas do roteiro da entrevista semiestrutura \\
\hline 1. Qual o envolvimento da sua família durante a fase inicial da sua carreira desportiva? \\
2. Quem o influenciou a ingressar na natação? \\
3. Você teve algum apoio para não abandonar a carreira? \\
4. Seus pais (ou irmãos) praticavam alguma modalidade esportiva? \\
5. Que tipo de apoio sua família oferecia no início de sua carreira?
\end{tabular}


A duração média das entrevistas foi de 60 minutos, sendo que as mesmas foram gravadas e transcritas (Patton, 2002; Côté, Salmela, Baria, \& Russel, 1993; Moraes, 1999; Martens, 1987; Thomas, Nelson, \& Silverman, 2007; Jonhson et. al., 2008). As entrevistas foram transcritas por meio de digitação simultânea das gravações (Patton, 2002; Martens, 1987; Johnson et al., 2008). Foi realizada a descontextualização das mesmas, ou seja, todas as perguntas do pesquisador foram retiradas, restando apenas uma narrativa única do entrevistado (Strean, 1998).

As entrevistas foram enviadas aos voluntários para uma confirmação do que havia sido transcrito, juntamente com uma carta-resposta para ser assinada, confirmando a veracidade dos dados (Krefiting, 1991; Patton, 2002). O texto foi considerado fidedigno pelos entrevistados, a partir de uma carta-resposta, assinada por todos os voluntários. Caso houvesse alguma correção, esta era realizada de acordo com as sugestões enviadas pelos voluntários.

\section{Análise dos Dados}

As narrativas foram categorizadas e analisadas por meio de Meaning Units (MiniUnidades - MUs) (Côté, et al., 1993; Johnson, et. al., 2008). Uma Meaning Unit (MU) representa uma parte do texto, que pode ser uma linha, parágrafo ou mais de um parágrafo do corpo de um texto, que exemplifica uma ideia expressada pelo pesquisado de forma clara e objetiva. Neste estudo, foram categorizadas e sub categorizadas de acordo com as similaridades identificadas entre as diversas MU's estabelecidas no estudo.

Essas MU's foram avaliadas por três expert em pesquisa qualitativa com o objetivo de validar os critérios usados para identificação de MU ou grupos de MUs. Após esta avaliação, as MU's foram subdivididas em categorias e sub categorias estabelecidas como representativas dos fatores investigados. A análise dos três expert mais uma vez se fez necessária para que, posteriormente, fosse realizada a interpretação dos dados (Côté, et al., 2005). As avaliações realizadas pelos voluntários e três expert em pesquisa qualitativa tiveram como objetivo aumentar a credibilidade/legitimidade exigidas na pesquisa qualitativa (Mathinson, 1988).

\section{RESULTADOS E DISCUSSÃO}

Após a transcrição das entrevistas, o que originou 73 páginas digitadas com espaçamento 1,5, fonte Times New Roman tamanho 12, procedeu-se a análise das MUs mais significativas. 127 MUs foram selecionadas, gerando um grupo de duas categorias, 5 subcategorias e duas propriedades (ver Figura 1), distribuídas dedutivamente para análise (Strauss \& Corbin, 1990).

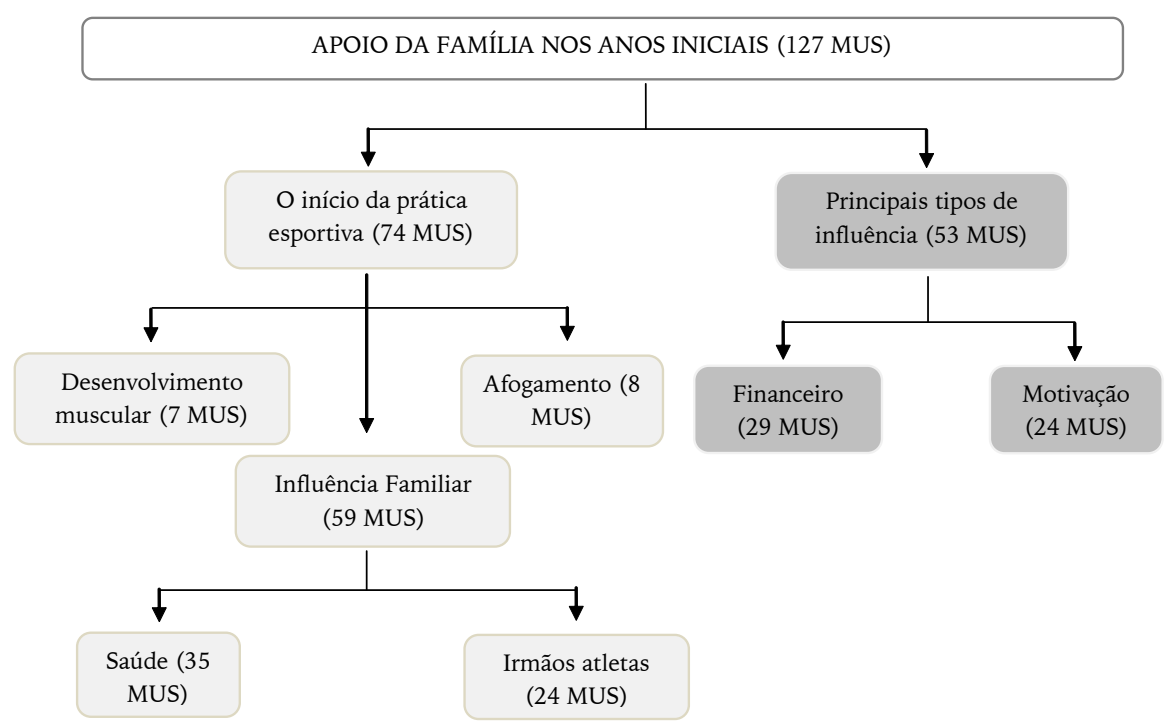

Figura 1. Distribuição das MUs em categorias, subcategorias e propriedades 


\section{Início da prática desportiva}

Os motivos pelos quais uma criança inicia a prática de uma modalidade desportiva são fundamentais para o progresso na mesma, pois contribuem para a sua permanência/persistência no desporto. Este início é advindo da influência direta da família (Côté, 1999). Os principais motivos que levaram os medalhistas ao início da prática da natação estão apresentados na Tabela 2:

\section{Tabela 2}

Motivos do início da prática da natação pelos nadadores medalhistas olímpicos

\begin{tabular}{cl}
\hline Atletas & Início da prática \\
\hline A1 & Desenvolvimento muscular \\
A2 & Irmãos atletas \\
A3 & Prática de vários esportes \\
A4 & Afogamento \\
A5 & Influência da família \\
A6 & Irmãos atletas \\
A7 & Problemas respiratórios/Influência da \\
A8 & família \\
\hline
\end{tabular}

Os resultados demonstram que, na fase de iniciação, a grande maioria dos nadadores teve a proximidade da família oferecendo apoio e incentivo para a manutenção da prática esportiva. Tal fator é designado como crítico por Moraes et al. (2004a) no decorrer do processo de aprendizagem do início da prática desportiva. Durand-Bush, Salmela e Thompson (2005) e Samulski et al. (2009), baseados na percepção de ex-atletas, verificaram também que os familiares foram os principais motivadores para o início da carreira esportiva dos mesmos.

De acordo Bloom (1985), durante os anos iniciais, os pais são os responsáveis por inserir os seus filhos numa atividade desportiva, além de prover apoio emocional e financeiro para que não ocorra o abandono da modalidade por falta desses incentivos (Falk et al., 2004). No presente estudo, os nadadores (A2, A3, A5, A6, A7, A8) apontaram que o principal motivo para a iniciação esportiva foi a influência direta da família, e a dos irmãos que praticavam a modalidade. Justificaram, ainda, que os pais os incentivaram por serem igualmente praticantes de modalidades desportivas, o que é exemplificado pela narrativa do atleta A2.

"Bem, o meu envolvimento aconteceu porque eu venho de uma família de atletas, de nadadores, meus três irmãos nadaram, eu sou o quarto e mais novo. Meu pai é um entusiasta muito grande em todos os esportes, principalmente na natação. Começou com meu irmão mais velho que era asmático e o médico sugeriu que ele fizesse um esporte e indicou a natação. Aí meu pai matriculou o A. na natação e daí ele se envolveu, gostou demais e depois do A. veio o R., veio o R., depois veio eu." (A2) - Início e influência da família

No entanto, os atletas A1 e A4 tiveram como principal motivo de iniciação na natação o desenvolvimento muscular e o acometimento de um afogamento. Esses fatos motivaram os familiares destes atletas a ingressarem na natação, o que não deixou de ser uma influência familiar indireta.

Para Côté (1999), nos anos de experimentação, a inserção em diversas modalidades desportivas é de suma importância, pois desenvolve o gosto pela atividade desportiva, aprimorando a experiência motora do atleta e possibilitando, mais tarde, o mesmo a se especializar numa única modalidade. Este argumento pode ser observado pela narrativa do nadador A3.

"... meus pais decidiram que eu precisava realmente saber nadar. Aí na época eu comecei a fazer natação e judô. Foram as duas modalidades. E obviamente eu comecei a me destacar já na natação com mais sucesso, logo abandonei o judô e me dediquei só a natação." (A4) - Início generalizado

Estas narrativas estimulam a reflexão de que os pais, esta força invisível, que não aparecem no cenário de sucesso de seus filhos, são o elo entre os atletas e o percurso para atingirem altos níveis de desempenho. Moraes (1999) 
encontrou resultados idênticos quando pesquisou judocas de nível internacional no Canadá.

\section{Principais tipos de influências}

O tipo e a constância do apoio proporcionado pela família (Tabela 3) foi também um dos norteadores da manutenção dos participantes na natação. Sem o apoio motivacional e financeiro, os filhos não teriam condições de frequentarem um clube. Segundo os nadadores, este tipo de apoio foi durante o início, meio e ápice das suas carreiras desportivas.

O tipo de apoio ofertado pelos pais no início e durante a carreira dos atletas influiu no desenvolvimento da criança no desporto. Este tipo de apoio que se manteve, para a maioria dos nadadores, refletiu na motivação para permanecer na modalidade, assim como no comprometimento com a carreira e a prática, o que corroborou com os estudos de Vernacchia et al. (2000), Durand-Bush e Salmela (2002) e Mallet e Hanrahan (2004).

A característica dessas famílias foi o envolvimento, dos pais apoiando os filhos de forma adequada, sem sobre ou sub valorizar seu comprometimento, confirmando Hellstedt (1987). O apoio da família dos nadadores durante este período foi evidenciado, por meio do apoio emocional e financeiro, confirmando Moraes et al. (2004b) e Samulski et al. (2009), que destacam a importância do apoio da família para a permanência dos seus filhos na modalidade esportiva.

Outro importante ponto a se destacar é que estas famílias podem ser caracterizadas como famílias complexas (Czikszentmihalyi, et al., 1993), já que elas transmitem segurança não somente a partir de incentivos financeiros, mas também motivacionais, estimulando os seus filhos a procurarem por desafios, como o apoio para o treino fora do país ou por melhores locais de treino, corroborando com os achados de Salmela e Moraes (2003). Tal fato pode ser elucidado pela MU do atleta A5.

"... normalmente a família veste a vontade no atleta. Eles são incentivadores porque levam os atletas para treinar, eles dão suporte... e toda competição que era importante, eles iam lá e presenciavam. Mas não me cobravam diretamente." (A5) - Apoio da família

Para o nadador A8, a família, no ápice de sua carreira, exerceu a função de gerência, deixando para o filho somente a preocupação de treinar, toda estrutura existente, como transporte, alimentação, parte financeira, entre outras era de responsabilidade dos pais, promovendo, dessa forma, todas as facilidades para a melhora do desempenho do atleta.

Tabela 3

Tipo de apoio recebido pelo atleta durante a carreira, advindo da família

\begin{tabular}{cccc}
\hline \multirow{2}{*}{ Atletas } & Início & Apoio da família durante a carreira & \\
& Financeiro/Motivação & Financeiro/Motivação & Ainance \\
\hline A1 & Financeiro/Motivação & Financeiro/Motivação & Motivaçããa \\
A2 & Financeiro/Motivação & Financeiro/Motivação & Motivação \\
A3 & Financeiro/Motivação & Financeiro/Motivação & - \\
A4 & Financeiro/Motivação & Financeiro/Motivação & Motivação \\
A5 & Financeiro/Motivação & Motivação & Motivação \\
A6 & Financeiro/Motivação & Motivação & Motivação \\
A7 & Financeiro/Motivação & Financeiro/Motivação & Gerenciamento/Motivação \\
A8 & & &
\end{tabular}


"Com 13 anos eu comecei a perder, foi difícil. Meus pais me seguraram. E aí depois quando eu vim para São Paulo, fui para os EUA, todo esse backstage, todo esse cenário atrás que ninguém vê, escolher o melhor ponto no apartamento, para estar perto do clube, a pé, na frente do colégio, estar junto das coisas, eu nunca corri atrás, foi sempre meu pai e minha mãe que fizeram tudo. Eu só chegava e tinha que nadar mesmo, o que eu sempre fiz foi chegar, treinar e nadar, fazer o que eu tinha que fazer." (A8) - Apoio da família

Diante dos dados apresentados nesta pesquisa, mais uma vez, confirma-se que a presença familiar, constante, silenciosa, fora de foco da mídia, contribui marcantemente para a formação expert destes nadadores medalhistas olímpicos.

Este trabalho representa um levantamento histórico acerca da influência que as famílias dos medalhistas olímpicos da natação brasileira tiveram em seu desenvolvimento desportivo. Contudo, o estudo contém algumas limitações: o número reduzido da amostra; a análise de diferentes gerações esportivas; e o método empregado, no caso uma entrevista. Em relação ao método, a fidedignidade das informações depende, diretamente, da motivação, honestidade, memória e capacidade de resposta dos desportistas.

\section{CONCLUSÕES}

A partir das ponderações apresentadas neste estudo, conclui-se que a influência da família em todas as fases, do início ao ápice, contribuiu de forma fundamental para o desenvolvimento dos atletas. Destaca-se, contudo, a primeira fase como especial, representado pelo apoio familiar, marcado por apoio financeiro e motivacional e a sustentação para o prosseguimento dos nadadores no desporto.

O início da carreira dos atletas foi de fundamental importância para seu desenvolvimento posterior. A família esteve presente direta ou indiretamente, influenciando a criança a iniciar e a permanecer na natação. A contribuição mais encontrada para a iniciação da prática da modalidade foi a influência de irmãos e/ou pais atletas, praticantes de desporto. $\mathrm{O}$ apoio financeiro e motivacional também contribuiu para o desenvolvimento do atleta e para a permanência das crianças nos anos iniciais da prática da natação.

A investigação da influência da família na primeira fase no contexto de medalhistas olímpicos se fez justificável, pois, existe uma carência de estudos, principalmente analisando o contexto brasileiro, sobre a influência da família, especialmente no nível destes nadadores olímpicos. Sugere-se a realização de mais estudos analisando outras modalidades, no entanto, mantendo-se os critérios de inclusão, com o objetivo de se descrever a trajetória de outros atletas olímpicos no Brasil.

\section{Agradecimentos:}

Os autores agradecem o apoio concedido pela FAPEMIG e Confederação Brasileira de Desportos Aquáticos (CBDA) para a realização do presente estudo.

\section{Conflito de Interesses:}

Nada a declarar.

\section{Financiamento:}

Nada a declarar.

\section{REFERÊNCIAS}

Baker, J., \& Horton, S. (2004). A review of primary and secondary influences on sport expertise. High Ability Studies, 15(2), 211-228. doi: 10.10 80/1359813042000314781

Bloom, B. S. (1985). Developing talent in young people. New York, EUA: Ballantine Books.

Côté, J. (1999). The influence of the family in the development of talent in sport. The Sport Psychologist, 13(4), 395-417.

Côté, J., Ericsson, K. A., \& Law, M. P. (2005). Tracing the development of athletes using retrospective interview methods: A proposed interview and validation procedure for reported information. Journal of Applied Sport Psychology, 17(1), 1-19. doi: 10.1080/10413200590907531

Côté, J., Lidor, R., \& Hackfort, D. (2009). To sample or to specialize? Seven postulates about youth 
sport activities that lead to continued participation and elite performance. International Journal of Sport and Exercise Psychology, 7, 7-17.

Côté, J., Salmela, J. H., Baria, A., \& Russel, S. J. (1993). Organizing and interpreting unstructured qualitative data. The Sport Psychologist, 7(2), 127-137.

Csikszentmihalyi, M., Rathunde, K., \& Whalen, S. (1993). Talent teenagers: The root of success and failure. Cambridge, UK: Cambridge University Press.

Durand-Bush, N., \& Salmela, J. H. (2002). The development and maintenance of expert athletic performance: Perceptions of world and Olympic champions. Journal of Applied Sport Psychology, 14(3), 154-171. doi: 10.1080/10413 200290103473

Durand-Bush, N., Salmela, J. H., \& Thompson, K. A. (2005). The role of parents in the development and maintenance of expertise in sport. In $\mathrm{T}$. Morris, P. Terry, S. Gordon, S. Hanrahan, L. Ievleva, G. Kolt, \& P. Tremayne (Eds.), Proceedings of the ISSP 11th World Congress of Sport Psychology. Sydney, Australia: International Society of Sport Psychology (ISSP).

Ericsson, K. A, Prietula, M. J., \& Cokely, E. T. (2007). The making of an expert. Harvard Business Review, Jul/Aug, 18-26.

Ericsson, K. A.; Lehmann, A. C. (1996). Expert and exceptional performance: Evidence of maximal adaptation to task constraints. Annual Reviews Psychology, 47(1), 273-305.

Falk, B., Lidor, R., Landre, Y., \& Lang, B. (2004). Talent identification and early development of elite water-polo players: A 2-year follow-up study. Journal of Sports Sciences, 22 (4), 347-355. doi: 10.1080/02640410310001641566

Gustafsson, H., Kentta, G., Hassmén, P., \& Lundqvist, C. (2007). Prevalence of burnout in competitive adolescent athletes. The Sport Psychologist, 21 (1), 21-37.

Hellstedt, J. C. (1987). The coach/parent/athlete relationship. The Sport Psychologist, 1(2), 151160.

Johnson, M. B., Castillo, Y., Sacks, D. N., Cavazos Jr. J., Edmonds, W. A., \& Tenenbaum, G. (2008). Hard work beats talent until talent decides to work hard: Coaches' perspectives regarding differentiating elite and non-elite swimmers. International Journal of Sports Science $\mathcal{E}$ Coaching, 3(3), 417-430. doi: 10.1260/1747954 08786238579
Krefting, L. (1991). Rigor in qualitative research: The assessment of trustworthiness. The American Journal of Occupational Therapy, 45, 214-222.

Mallet, C. J., \& Hanrahan, S. J. (2004). Elite athletes: Why does the 'fire' burn so brightly. Psychology of Sport and Exercise, 5(2), 183-200. doi: 10.1016/S1469-0292(02)00043-2

Marques, M., \& Samulski, D. (2009). Análise da carreira esportiva de jovens atletas de futebol na transição da fase amadora para a fase profissional: Escolaridade, iniciação, contexto sóciofamiliar e planejamento da carreira. Revista Brasileira de Educação Física e Esporte, 23(2), 103-119.

Martens, R. (1987). Science, knowledge, and sport psychology. The Sport Psychologist, 1 (1), 29-55.

Mathinson, S. (1988). "Why triangulate?". Educational Researcher, 17(2), 13-17. doi: 10.3102/00 13189X017002013

McCarthy, P. J., \& Jones, M. V. (2007). A qualitative study of sport enjoyment in the sampling years. The Sport Psychologist, 21 (4), 400-416.

Moraes, L. C. (1999). Influences on the development of beliefs of Canadian expert judo coaches and their impact on action (Unpublished doctoral dissertation). University of Ottawa, Canada.

Moraes, L. C., \& Sousa, C. D. (2004). As diferentes influências da tríade pais-atletas-treinadores na trajetória de desenvolvimento de judocas brasileiros de nível internacional. In E. S. Garcia \& K. L. Lemos (Eds.), Temas Atuais em Educação Física e Esporte (pp. 111-132), Belo Horizonte: Editora Gráfica Silveira.

Moraes, L. C., Rabelo, A. S., Salmela, J. H., Lima, O. M., \& Lôbo, I. L. (2001). Desenvolvimento de jovens atletas de voleibol. Comunicação apresentada no VIII Congresso Brasileiro de Psicologia do Esporte, Belo Horizonte: CENESP.

Moraes, L. C., Rabelo, A. S., \& Salmela, J. H. (2004a). Papel dos pais no desenvolvimento de jovens futebolistas. Psicologia: Reflexão e Crítica, 17(2), 211-222.

Moraes, L. C., Salmela, J. H., Rabelo, A. S., \& Vianna Jr, N. S. (2004b). Le rôle des parents dans le développement des jeunes joueurs de football et de tennis brésiliens. Revue International des Sciences du Sport et de l'Education PhysiqueStaps, 25(64), 109-126.

Morgan, T. K., \& Giacobbi Jr., P. R. (2006). Toward two grounded theories of the talent development and social support process of highly successful collegiate athletes. The Sport Psychologist, 20(1), 295-313. 
Patton, M. (2002). Qualitative evaluation methods ( $3^{\mathrm{a}}$ ed.). California, EUA: Sage.

Salmela, J. H., \& Moraes, L. C. (2003). Development of Expertise: The role of coaching, families and cultural contexts. In J. L. Starkes \& K. A. Ericsson (Eds.), Expert performance in sports (pp. 272291), Champaign, Il: Human Kinetics.

Salmela, J. H., Young, B. W., \& Kallio, J. (2000). Within-career transitions of the athlete-coachparent triad. In D. Lavallee \& P. Wylleman (Eds.), Career transitions in sport: International perspectives (pp. 181-193). Morgantown, WV: Fitness Information Technology.

Samulski, D., Moraes, L., Ferreira, R. M., Marques, M., Silva, L. A., Lôbo, I., ... Ferreira, C. H. (2009). Análise das transições das carreiras de ex-atletas de alto nível. Motriz, 15(2), 310-317.

Strauss, A., \& Corbin, J. (1990). Basics of qualitative research: Grounded theory procedures and techniques. Newbury Park, CA: Stage.
Strean, W. B. (1998). Possibilities for qualitative research in sport psychology. The Sport Psychologist, 12(3), 333-345.

Tesch, R. (1990). Qualitative research: Analysis types and software tools. New York: Falmer Press.

Thomas, J. R., Nelson, J. K., \& Silverman, S. J. (2007). Métodos de pesquisa em atividade física (5 ed.). Porto Alegre: Artmed.

Vernacchia, R. A., McGuire, R. T., Reardon, J. R., \& Templin, D. P. (2000). Psychosocial characteristics of Olympic track and field athletes. International Journal of Sport Psychology, 31(1), 523.

Vianna Jr., N. S., Moraes, L. C., Salmela, J. H., \& Mourthé, K. (2001). The role of parents in the development of young Brazilian athletes in rhythmic gymnastics. Comunicação apresentada na $16^{\text {th }}$ Annual Conference of the Association for Advancement of Applied Psychology, Texas, USA, 1, 61 .

(c) EY-No quando especificado em contrário e nos conteúdos retirados de outras fontes bibliográficas. 